\title{
Fixed points and orbits of non-convolution operators
}

\author{
Fernando León-Saavedra ${ }^{1 *}$ and Pilar Romero-de la Rosa²
}

"Correspondence:

fernando.leon@uca.es

'Department of Mathematics, University of Cádiz, Avda. de la

Universidad s/n, Jerez de la Frontera, Cádiz 11405, Spain

Full list of author information is available at the end of the article

\begin{abstract}
A continuous linear operator $T$ on a Fréchet space $F$ is hypercyclic if there exists a vector $f \in F$ (which is called hypercyclic for $T$ ) such that the orbit $\left\{T^{n} f: n \in \mathbb{N}\right\}$ is dense in $F$. A subset $M$ of a vector space $F$ is spaceable if $M \cup\{0\}$ contains an infinite-dimensional closed vector space. In this paper note we study the orbits of the operators $T_{\lambda, b} f=f^{\prime}(\lambda z+b)(\lambda, b \in \mathbb{C})$ defined on the space of entire functions and introduced by Aron and Markose (J. Korean Math. Soc. 41(1):65-76, 2004). We complete the results in Aron and Markose (J. Korean Math. Soc. 41(1):65-76, 2004), characterizing when $T_{\lambda, b}$ is hypercyclic on $H(\mathbb{C})$. We characterize also when the set of hypercyclic vectors for $T_{\lambda, b}$ is spaceable. The fixed point of the map $z \rightarrow \lambda z+b$ (in the case $\lambda \neq 1$ ) plays a central role in the proofs.
\end{abstract}

Keywords: fixed point; Denjoy-Wolf theorem; non-convolution operator; hypercyclic operator; spaceability

\section{Introduction}

Let us denote by $F$ a complex infinite dimensional Fréchet space. A continuous linear operator $T$ defined on $F$ is said to be hypercyclic if there exists a vector $f \in F$ (called hypercyclic vector for $T)$ such that the orbit $\left(\left\{T^{n} f: n \in \mathbb{N}\right\}\right)$ is dense in $F$. We refer to the books $[1,2]$ and the references therein for further information on hypercyclic operators. From a modern terminology, a subset $M$ of a vector space $F$ is said to be spaceable if $M \cup\{0\}$ contains an infinite-dimensional closed vector space. The study of spaceability of (usually pathological) subsets is a natural question which has been studied extensively (see [1] Chapter 8 or the recent survey [3] and the references therein).

In 1991, Godefroy and Shapiro [4] showed that every continuous linear operator $L$ : $H(\mathbb{C}) \rightarrow H(\mathbb{C})$ which commutes with translations (these operators are called convolution operators) and which is not a multiple of the identity is hypercyclic. This result unifies two classical results by Birkhoff and MacLane (see the survey [5]).

In [5], Aron and Markose introduced new examples of hypercyclic operators on $H(\mathbb{C})$ which are not convolution operators. Namely, $T_{\lambda, b} f=f^{\prime}(\lambda z+b), \lambda, b \in \mathbb{C}$. In the first section we show that if $\lambda \in \mathbb{D}$ and $b \in \mathbb{C}$ then $T_{\lambda, b}$ is not hypercyclic on $H(\mathbb{C})$. This result together with the results in [5] and [6] shows the following characterization: $T_{\lambda, b}$ is hypercyclic on $H(\mathbb{C})$ if and only if $|\lambda| \geq 1$. Thus, we complete the results of Aron and Markose [5] and Fernández and Hallack [6] characterizing when $T_{\lambda, b}(\lambda, b \in \mathbb{C})$ is hypercyclic. Let us denote by $H C(T)$ the set of hypercyclic vectors for $T$. In Section 3 we characterize when $H C\left(T_{\lambda, b}\right)$ 
is spaceable. Namely $H C\left(T_{\lambda, b}\right)$ is spaceable if and only if $|\lambda|=1$. During the proofs, it is essential to take into account the fixed point of the map $z \rightarrow \lambda z+b(\lambda \neq 1)$.

\section{Characterizing the hypercyclicity of $T_{\lambda, b}$}

The proof of this result follows the ideas of the proof of Proposition 14 in [5].

Theorem 2.1 For any $\lambda \in \mathbb{D}$ and $b \in \mathbb{C}$ and for any $f \in H(\mathbb{C})$, the sequence $T_{\lambda, b}^{n} f \rightarrow 0$ uniformly on compact subsets of $\mathbb{C}$. Therefore $T_{\lambda, b}$ is not hypercyclic on $H(\mathbb{C})$.

Proof Set $\varphi(z)=\lambda z+b, \lambda \in \mathbb{D}$ and $b \in \mathbb{C}$. Since $\lambda \neq 1, \varphi(z)$ has a fixed point $z_{0}$. Indeed, $z_{0}=\frac{b}{1-\lambda}$. We denote by $\varphi_{n}(z)$ the sequence of the iterates defined by

$$
\varphi_{n}(z)=\varphi \circ \cdots \circ \varphi \quad(n \text { times }),
$$

an easy computation yields

$$
\varphi_{n}(z)=\lambda^{n} z+\frac{1-\lambda^{n}}{1-\lambda} b
$$

Let us observe that the iterates of the operator $T_{\lambda, b}$ have the form

$$
T_{\lambda, b}^{n} f(z)=\lambda^{\frac{n(n-1)}{2}} f^{(n)}\left(\lambda^{n} z+\frac{\left(1-\lambda^{n}\right) b}{1-\lambda}\right)=\lambda^{\frac{n(n-1)}{2}} f^{(n)}\left(\varphi_{n}(z)\right),
$$

where $f^{(n)}$ denotes the $n$th derivative of $f$. It is well known that if $\lambda \in \mathbb{D}$ then $z_{0}$ is an attractive fixed point, that is, $\varphi_{n}(z)$ converges to the fixed point $z_{0}$ uniformly on compact subsets. Indeed, let $R>0$. If $|z| \leq R$, then

$$
\left|\varphi_{n}(z)-z_{0}\right|=\left|\lambda^{n} z+\frac{\left(1-\lambda^{n}\right) b}{1-\lambda}-\frac{b}{1-\lambda}\right| \leq|\lambda|^{n} R+\frac{|\lambda|^{n}}{|1-\lambda|}|b| \rightarrow 0
$$

as $n \rightarrow \infty$. Thus, there exists $n_{0}$ such that if $|z| \leq R$ then $\left|\varphi_{n}(z)-z_{0}\right|<1 / 2$ for all $n \geq n_{0}$.

If $n \geq n_{0}$ and $|z| \leq R$, we have by the Cauchy inequality

$$
\left|f^{(n)}\left(\varphi_{n}(z)\right)\right| \leq C n ! 2^{n}, \quad \text { where } C=\max \{|f(w)|:|w| \leq 1\} .
$$

Now, it follows from Stirling's formula that $n ! \leq e n^{n+1 / 2} e^{-n}$. Hence, if $|z| \leq R$ and $n \geq n_{0}$, then

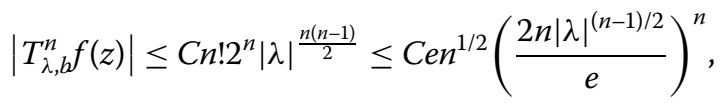

and since $2 n|\lambda|^{(n-1) / 2} \rightarrow 0$ as $n \rightarrow \infty$, we conclude that $\max _{|z| \leq R}\left|T_{\lambda, b}^{n} f(z)\right| \rightarrow 0$, as $n \rightarrow \infty$, as desired. We point out that this is a refinement of the argument by Aron and Markose. One of the referees chased the constants and recovered the factor $n^{1 / 2}$ that was missing but that does not break the argument.

Theorem 13 in [5] and Theorem 2.1 give the following characterization.

Theorem 2.2 For any $\lambda \in \mathbb{C}$ and $b \in \mathbb{C}$, the operator $T_{\lambda, b}$ is hypercyclic in $H(\mathbb{C})$ if and only if $|\lambda| \geq 1$. 


\section{Spaceability of the set of hypercyclic vectors for $T_{\lambda, b}$}

As stated in [3], there are few non-trivial examples of subsets $M$ which are lineable (that is, $M \cup\{0\}$ contains an infinite-dimensional vector space) and are not spaceable. The following result provides the following examples: for $|\lambda|>1$, the set $H C\left(T_{\lambda, b}\right)$ is lineable but it is not spaceable.

Shkarin [7] showed that for the derivative operator $D$, the set of hypercyclic vectors $H C(D)$ is spaceable.

Theorem 3.1 For any $\lambda \in \mathbb{C}$ and $b \in \mathbb{C}, H C\left(T_{\lambda, b}\right)$ is spaceable if and only if $|\lambda|=1$.

Proof Firstly, let us suppose that $|\lambda|>1$, and let us prove that $H C\left(T_{\lambda, b}\right)$ does not contain a closed infinite dimensional subspace. Let $z_{0}$ be the fixed point of $\varphi(z)=\lambda z+b$. Then we consider a sequence of norms defining the topology of $H(\mathbb{C})$. Namely, for $n \in \mathbb{N}$ and $f \in H(\mathbb{C})$, we write

$$
p_{n}(f)=\max _{\left|z-z_{0}\right| \leq|\lambda|^{n / 4}}|f(z)| .
$$

It is easy to see that the above sequence of semi-norms is increasing and defines the original topology on $H(\mathbb{C})$.

Given the sequence of increasing semi-norms $\left\{p_{n}\right\}$, according to Theorem 10.25 in [2], it is sufficient to find a sequence of subspaces $M_{n} \subset H(\mathbb{C})$ of finite codimension, positive numbers $C_{n} \rightarrow \infty$ and $N \geq 1$ satisfying the following:

(a) $p_{N}(f)>0, \forall f \in H C\left(T_{\lambda, b}\right)$.

(b) $p_{N}\left(T_{\lambda, b}^{n} f\right) \geq C_{n} p_{n}(f), \forall f \in M_{n}$.

Indeed, let us consider the subspaces

$$
M_{n}=\left\{f \in H(\mathbb{C}): f\left(z_{0}\right)=f^{\prime}\left(z_{0}\right)=\cdots=f^{(n-1)}\left(z_{0}\right)=0\right\},
$$

which are clearly of finite codimension.

Notice that $\varphi_{n}(z)-z_{0}=\lambda^{n}\left(z-z_{0}\right)$, so that $\varphi_{n}(z)$ maps the disk $D\left(z_{0}, 1\right)=\left\{\left|z-z_{0}\right| \leq 1\right\}$ onto $D\left(z_{0}, \|\left.\lambda\right|^{n}\right)$. Hence,

$$
\begin{aligned}
p_{0}\left(T_{\lambda, b}^{n} f\right) & =\max _{\left|z-z_{0}\right| \leq 1}\left|T_{\lambda, b}^{n} f(z)\right| \\
& =|\lambda|^{\frac{n(n-1)}{2}} \max _{\left|z-z_{0}\right| \leq 1}\left|f^{(n)}\left(\varphi_{n}(z)\right)\right| \\
& =|\lambda|^{\frac{n(n-1)}{2}} \max _{\left|\varphi_{n}(z)-z_{0}\right| \leq|\lambda|^{n+1}}\left|f^{(n)}\left(\varphi_{n}(z)\right)\right| \\
& =|\lambda|^{\frac{n(n-1)}{2}} \max _{\left|w-z_{0}\right| \leq|\lambda|^{n}}\left|f^{(n)}(w)\right| .
\end{aligned}
$$

If $f \in M_{1}$ then $f\left(z_{0}\right)=0$, so that $f(z)=\int_{\left[z_{0}, z\right]} f^{\prime}(\xi) d \xi$. Therefore we have

$$
\max _{\left|z-z_{0}\right| \leq R}|f(z)| \leq R \max _{\left|z-z_{0}\right| \leq R}\left|f^{\prime}(z)\right|,
$$

and it follows easily by induction that if $f \in M_{n}$ then

$$
\max _{\left|z-z_{0}\right| \leq R}|f(z)| \leq R^{n} \max _{\left|z-z_{0}\right| \leq R}\left|f^{(n)}(z)\right| .
$$


Thus,

$$
\begin{aligned}
p_{0}\left(T_{\lambda, b}^{n} f\right) & =|\lambda|^{\frac{n(n-1)}{2}} \max _{\left|w-z_{0}\right| \leq|\lambda|^{n}}\left|f^{(n)}(w)\right| \\
& \geq|\lambda|^{\frac{n(n-1)}{2}} \max _{\left|w-z_{0}\right| \leq|\lambda|^{n / 4}}\left|f^{(n)}(w)\right| \\
& \geq|\lambda|^{\frac{n(n-1)}{2}}|\lambda|^{-n^{2} / 4} \max _{\left|w-z_{0}\right| \leq|\lambda|^{n / 4}}|f(w)| \\
& =|\lambda|^{\frac{n^{2}-2 n}{4}} p_{n}(f),
\end{aligned}
$$

and it follows that condition (b) is satisfied with $N=0$ and $C_{n}=|\lambda|^{\frac{n^{2}-2 n}{4}} \rightarrow \infty$ as $n \rightarrow \infty$, and therefore $H C\left(T_{\lambda, b}\right)$ is not spaceable.

Now, let us suppose that $|\lambda|=1$, and let us prove that $H C\left(T_{\lambda, b}\right)$ is spaceable. Indeed, let us suppose first that $\lambda=1$. If $b=0$ then $T_{1,0}=D$, and it was proved by Shkarin [7] that $H C(D)$ is spaceable. If $b \neq 0$ then $T_{1, b}=D e^{b D}$, so that $T_{1, b}=\psi(D)$, where $\psi(z)=z e^{b z}$ is an entire function of exponential type that is not a polynomial, and according to Example 10.12 in [2, p.275], the space $H C\left(T_{1, b}\right)$ is spaceable.

Now let us consider the case $\lambda \in \partial \mathbb{D} \backslash\{1\}$. Set $z_{0}=\frac{b}{1-\lambda}$ the fixed point of $\varphi(z)=\lambda z+b$. According to Theorem 10.2 in [2], since $T_{\lambda, b}$ satisfies the hypercyclicity criterion for the full sequence of natural numbers, it suffices to exhibit an infinite dimensional closed subspace $M_{0}$ of $H(\mathbb{C})$ on which suitable powers of $T_{\lambda, b}$ tend to 0 . Now the proof mimics some ideas contained in Example 10.13 in [2]. Indeed, for any $n \geq 1$, there is some $C_{n}>0$ such that

$$
x^{n} \leq 2^{x} \text { for all } x \geq C_{n} .
$$

Let us consider a strictly increasing sequence of positive integers $\left(n_{k}\right)_{k}$ satisfying $n_{k+1} \geq$ $C_{n_{k}}$. If $j \geq k+1$, then $n_{j} \geq n_{k+1} \geq C_{n_{k}}$, therefore by (1) we have

$$
n_{j}^{n_{k}} \leq 2^{n_{j}} \quad \text { for } j \geq k+1
$$

Let us consider $M_{0}$ the closed subspace of $H(\mathbb{C})$ of all entire functions $f$ of the form

$$
f(z)=\sum_{k=1}^{\infty} a_{k}\left(z-z_{0}\right)^{n_{k}-1}
$$

and let us prove that $T_{\lambda, b}^{n_{k}} f \rightarrow 0$ uniformly on compact subsets as $k \rightarrow \infty$.

We have

$$
\left(T^{n_{k}} f\right)(z)=\lambda^{\frac{n_{k}\left(n_{k}-1\right)}{2}}\left(D^{n_{k}} f\right)\left(\varphi_{n_{k}}(z)\right)
$$

Notice that $|\lambda|=1$ and the map $\varphi_{n_{k}}$ takes the $\operatorname{disc} D\left(z_{0}, R\right)$ onto itself, so that

$$
\begin{aligned}
\max _{\left|z-z_{0}\right| \leq R}\left|\left(T^{n_{k}} f\right)(z)\right| & =\max _{\left|z-z_{0}\right| \leq R}\left|\left(D^{n_{k}} f\right)\left(\varphi_{n_{k}}(z)\right)\right| \\
& =\max _{\left|w-z_{0}\right| \leq R}\left|\left(D^{n_{k}} f\right)(w)\right| .
\end{aligned}
$$


Finally, we have

$$
\begin{aligned}
\max _{\left|w-z_{0}\right| \leq R}\left|\left(D^{n_{k}} f\right)(w)\right| & =\max _{\left|w-z_{0}\right| \leq R}\left|\sum_{j=k+1}^{\infty} a_{j} D^{n_{k}}\left(w-z_{0}\right)^{n_{j}-1}\right| \\
& \leq \sum_{j=k+1}^{\infty}\left|a_{j}\right|\left(n_{j}-1\right)\left(n_{j}-2\right) \cdots\left(n_{j}-n_{k}\right) R^{n_{j}-n_{k}-1} \\
& \leq \sum_{j=k+1}^{\infty}\left|a_{j}\right| n_{j}^{n_{k}} R^{n_{j}} \\
& \leq \sum_{j=k+1}^{\infty}\left|a_{j}\right|(2 R)^{n_{j}} \rightarrow 0 \quad \text { as } k \rightarrow \infty
\end{aligned}
$$

In the last step we used inequality (2). This completes the proof of Theorem 3.1.

\section{Competing interests}

The authors declare that they have no competing interests.

\section{Authors' contributions}

Both authors contributed equally in this article. They read and approved the final manuscript.

\section{Author details}

1Department of Mathematics, University of Cádiz, Avda. de la Universidad s/n, Jerez de la Frontera, Cádiz 11405, Spain.

2Department of Mathematics, IES Sofía, Doctor Marañón, 13, Jerez de la Frontera, Cádiz 11407, Spain.

\section{Acknowledgements}

The research was supported by Junta de Andalucía FQM-257. The authors would like to thank the referee for reading our manuscript carefully and for giving such constructive comments, which helped improving the quality of the paper substantially.

Received: 1 July 2014 Accepted: 14 October 2014 Published: 29 October 2014

\section{References}

1. Bayart, F, Matheron, É: Dynamics of Linear Operators. Cambridge Tracts in Mathematics, vol. 179, p. xiv+337. Cambridge University Press, Cambridge (2009). doi:10.1017/CBO9780511581113

2. Grosse-Erdmann, K-G, Peris Manguillot, A: Linear Chaos. Universitext, p. xii+386. Springer, London (2011). doi:10.1007/978-1-4471-2170-1

3. Bernal-González, L, Pellegrino, D, Seoane-Sepúlveda, JB: Linear subsets of nonlinear sets in topological vector spaces. Bull. Am. Math. Soc. (N.S.) 51(1), 71-130 (2014). doi:10.1090/S0273-0979-2013-01421-6

4. Godefroy, G, Shapiro, JH: Operators with dense, invariant, cyclic vector manifolds. J. Funct. Anal. 98(2), 229-269 (1991). doi:10.1016/0022-1236(91)90078-J

5. Aron, R, Markose, D: On universal functions. Satellite Conference on Infinite Dimensional Function Theory J. Korean Math. Soc. 41(1), 65-76 (2004). doi:10.4134/JKMS.2004.41.1.065

6. Fernández, G, Hallack, AA: Remarks on a result about hypercyclic non-convolution operators. J. Math. Anal. Appl. 309(1), 52-55 (2005). doi:10.1016/j.jmaa.2004.12.006

7. Shkarin, S: On the set of hypercyclic vectors for the differentiation operator. Isr. J. Math. 180, 271-283 (2010). doi:10.1007/s11856-010-0104-z 